\title{
WHO downgrades status of oseltamivir
}

The final paragraph of this editorial (BMJ 2017;358:j3266, doi:10.1136/bmj.j3266) contains an incorrect statement.
Oseltamivir was not removed from the essential medicine's list. The drug's status was downgraded. 